\title{
Higher BMP/Smad sensitivity of tendon-derived stem cells (TDSCs) isolated from the collagenase-induced tendon injury model: possible mechanism for their altered fate in vitro
}

Pauline Po Yee Lui ${ }^{1 *}$ and Yin Mei Wong ${ }^{2,3}$

\begin{abstract}
Background: Ectopic expression of BMP-2, BMP-4 and BMP-7 was observed in clinical samples of tendinopathy and collagenase-induced $(\mathrm{Cl})$ tendon injury rat model. TDSCs isolated from the $\mathrm{Cl}$ model showed increased non-tenogenic differentiation potential and hence altered fate compared to the TDSCs isolated from the healthy animals (HT) but the mechanism is unclear. We hypothesized that sensitization of the BMP/Smad pathway in TDSCs (Cl) might account for this difference. This study aimed to compare the activation state of the BMP/Smad pathway at basal level and upon BMP-2 stimulation in TDSCs (Cl) and TDSCs (HT).
\end{abstract}

Methods: Collagenase or saline was injected into the patellar tendon of rats for 2 weeks. TDSCs (CI) and TDSCs (HT) were then isolated from the patellar tendon. The mRNA and protein expression of BMPs and BMP receptors in TDSCs (CI) and TDSCs (HT) were analysed. TDSCs from both sources were treated with rhBMP-2 and the expression of phosphorylated and total Smad1/5/8 was examined.

Results: Except for the mRNA levels of Bmp7 and Bmpr2, there were significant higher mRNA and protein expression of BMPs and BMP receptors in TDSCs (Cl) compared to TDSCs (HT). TDSCs (Cl) showed higher basal expression of total Smad1/5/8 but similar basal level of phosphorylated Smad1/5/8 compared to TDSCs (HT). TDSCs (CI) exhibited higher total and phosphorylated Smad1/5/8 upon BMP-2 stimulation.

Conclusions: The sensitization of the BMP/Smad pathway in TDSCS (CI) might account for their higher non-tenogenic differentiation potential and hence altered fate. It also provided further support of BMPs and the $\mathrm{BMP} / \mathrm{Smad}$ signaling pathway in the pathogenesis of tendinopathy.

Keywords: Tendinopathy, Tendon-derived stem/progenitor cells, BMP/Smad signaling, Collagenase-induced tendon injury

\section{Background}

Tendinopathy is a chronic painful tendon disorder that is common among athletes and middle-aged people with repetitive tendon overuse. Histopathologically, tendinopathy is characterized by an increase of cellularity [1-3], vascularity [1], glycosaminoglycan deposition [1,2] and loss of matrix organization $[1,4]$. Tissue metaplasia, with the presence of chondrocyte phenotype [5] and occasional fatty and

\footnotetext{
* Correspondence: paulinelui00@gmail.com

'Headquarter, Hosptial Authority, 9/F, Rumsey Street Multi-Storey Carpark Building, Sheung Wan, Hong Kong SAR, China

Full list of author information is available at the end of the article
}

bony deposits $[2,4,5]$, is observed. The pathogenesis of tendinopathy remains unclear. As a result, only symptomatic treatments are currently provided with limited success $[6,7]$. The cells isolated from the tendinopathic tissue showed high metabolic activity [3]. Therefore, failed healing, rather than degeneration, is suggested as the pathogenesis of tendinopathy [8].

Ectopic expression of BMP-2, -4 and -7 were observed in clinical samples of tendinopathy $[9,10]$ and collagenaseinduced $(\mathrm{CI})$ tendon injury rat model which showed failed healing and ectopic chondro-ossification in tendons [11]. These BMPs were reported to promote osteogenic, 
chondrogenic and adipogenic differentiation of mesenchymal stem cells (MSCs) including tendon-derived stem cells (TDSCs) isolated from healthy tendons in vitro [12-16]. Tendinopathic Achilles tendons showed higher mRNA expression of chondro-osteogenic markers compared to healthy tendons [17]. Consistent with this previous finding [17], TDSCs isolated from the CI model (TDSCs (CI)) showed altered fate, with higher chondro-osteogenic differentiation potential but lower tenogenic marker expression compared to TDSCs isolated from the healthy animals (TDSCs (HT)) [18]. However, the mechanism of altered fate of TDSCs $(\mathrm{CI})$ is unclear. We hypothesized that the increased sensitivity of the BMP/Smad signaling pathway in TDSCs (CI) might account for their higher non-tenogenic differentiation potential and hence altered fate. This study therefore aimed to compare the activation state of the BMP/Smad pathway at basal level and upon BMP-2 stimulation in TDSCs isolated from CI model and healthy animals. Results from this study would provide novel insight of the pathogenic mechanism of tendinopathy.

\section{Methods}

\section{Collagenase-induced tendon injury model}

This study was approved by the Animal Research Ethics Committee of the authors' institution (Ref no: 10/010/ GRF). Twelve male Sprague Dawley rats, (6 weeks, weight 150-220 grams) were used. The procedures have been well-established and the histopathological changes were highly reproducible [19]. After anesthesia with $2.5 \%$ pentobarbital ( $4.5 \mathrm{mg} / \mathrm{kg}$ body weight), hairs over the lower limb were shaved. The patellar tendon was located by positioning the knee at $90^{\circ}$. Twenty microliters $(0.015 \mathrm{mg} / \mu \mathrm{l}$ in $0.9 \%$ saline, i.e. $0.3 \mathrm{mg}$ ) of bacterial collagenase I (Sigma-Aldrich, St Louis, MO, USA) (CI group) $(\mathrm{n}=6)$ or saline (HT group) $(n=6)$ were injected into both patellar tendons (i.e. both tendons were injected with collagenase or both tendons were injected with saline) of each rat intratendinously with a $30 \mathrm{G}$ needle. Free cage activity was allowed after injection. At week 2 after injection, rats were sacrificed and both patellar tendons of each rat were harvested and pooled together for the isolation of TDSCs (HT) or TDSCs (CI) [19]. Week 2 was chosen for the isolation of TDSCs (CI) when the direct effect of collagenase subsided and tendon healing with increase in cell proliferation occurred while no chondrocyte-like cells were observed at this time point [19].

\section{Isolation and culture of rat TDSCs}

The procedures for the isolation of TDSCs from the midsubstance of collagenase-injured and healthy patellar tendons have been established [20]. Briefly, after euthanasia, the mid-substances of both patellar tendons were excised. Care was taken that only the mid-substance of patellar tendon tissue, but not the tissue at the tendon-bone junction, was collected. The peritenon was carefully removed and the tissue was stored in sterile phosphate-buffered saline (PBS). The tissue was minced, digested with type I collagenase $(3 \mathrm{mg} / \mathrm{ml}$; Sigma-Aldrich, St Louis, MO, USA) and passed through a $70 \mu \mathrm{m}$ cell strainer (Becton Dickinson, Franklin Lakes, USA) to yield a single-cell suspension. The released cells were washed in PBS and resuspended in low glucose Dulbecco's Modified Eagle Medium (LG-DMEM) (Gibco BRL; Life Technologies, Invitrogen, Carlsbad, CA, USA), $10 \%$ fetal bovine serum (FBS), $50 \mu \mathrm{g} / \mathrm{ml}$ penicillin, $50 \mu \mathrm{g} / \mathrm{ml}$ streptomycin $100 \mu \mathrm{g} / \mathrm{ml}$ neomycin (complete culture medium) (all from Invitrogen corporation, Carlsbad, USA). The isolated nucleated cells were plated at an optimal low density $\left(50\right.$ cells $\left./ \mathrm{cm}^{2}\right)$ for the isolation of TDSCs from rat patellar tendon and cultured at $37^{\circ} \mathrm{C}, 5 \% \mathrm{CO}_{2}$ to form colonies. At day 2 after initial plating, the cells were washed twice with PBS to remove the non-adherent cells. At day 7-10, they were trypsinized and mixed together as passage 0 (P0). TDSCs were subcultured when they reached $80-90 \%$ confluence. The stem cell-related surface marker expression (including CD44, CD90 and CD73), clonogenicity and multi-lineage differentiation potential of the isolated nucleated cells from the CI animal model and healthy animals were confirmed as described previously before being used for the experiments in this study [18]. TDSCs (CI) and TDSCs (HT) at passage 5 were used for all the experiments.

\section{Study design}

TDSCs isolated from both sources were plated at 4000 cell $/ \mathrm{cm}^{2}$ in $100-\mathrm{mm}$ tissue culture dish and cultured in low glucose Dulbecco's Modified Eagle Medium (LGDMEM) (Gibco) supplemented with 10\% fetal bovine serum (FBS), $50 \mu \mathrm{g} / \mathrm{ml}$ penicillin, $50 \mu \mathrm{g} / \mathrm{ml}$ streptomycin and $100 \mu \mathrm{g} / \mathrm{ml}$ neomycin (complete culture medium) (all from Invitrogen corporation, Carlsbad, USA) at $37^{\circ} \mathrm{C}, 5 \%$ $\mathrm{CO}_{2}$ until confluence. The cells were then subjected to mRNA and protein analysis of expression of BMPs (BMP-2, BMP-4, BMP-7) and BMP receptors (BMPRIA, BMPR-IB, BMPR-II) using qRT-PCR and Western blotting (WB), respectively. To investigate the response of both cell types to BMP-2 stimulation, TDSCs (CI) and TDSCs (HT) isolated from each of three rats were plated at $4000 \mathrm{cell} / \mathrm{cm}^{2}$ in 6-wells plate and cultured in complete medium until the cells reached $80 \%$ confluence for immunocytochemical staining (ICC) and confluence for WB. They were then treated with rhBMP-2 $(100 \mathrm{ng} / \mathrm{ml})$ (Wyeth, Cambridge, MA, USA) in complete medium for $0,15,30$ and 60 minutes at $37^{\circ} \mathrm{C}, 5 \% \mathrm{CO}_{2}$. Time series data of TDSC (CI) and TDSC (HI) of each of 4 rats / group was obtained. The nuclear translocation of the phosphorylated form of Smad 1/5/8 (pSmad 1/5/8) was examined by ICC while the expression of the pSmad $1 /$ $5 / 8$ and total Smad 1/5/8 was examined by WB. BMP-2 
at $100 \mathrm{ng} / \mathrm{ml}$ was used in this study based on our previous study testing the effect of different concentrations of BMP-2 (0, 50, 100, 250, 500 and $1000 \mathrm{ng} / \mathrm{ml})$ and $100 \mathrm{ng} / \mathrm{ml}$ was the lowest dose that induced the osteogenic differentiation of TDSCs (unpublished results). BMP-2 at $100 \mathrm{ng} / \mathrm{ml}$ promoted non-tenogenic (osteo-, chondro- and adiop- genic) differentiation, increased proteoglycan production but inhibited tendon-related marker expression in TDSCs [13]. This same dose (100 ng/ml) was also used in a previous study investigating the effect of BMP-2 on the osteogenic response as well as the expression and translocation of pSmad 1/5/8 in tendon stem / progenitor cells [21].

\section{Quantitative real-time reverse transcription-polymerase chain reaction (qRT-PCR)}

Cells were harvested and homogenized for RNA extraction with Rneasy mini kit (Qiagen, Germany). The mRNA was reverse-transcribed to cDNA by the First Strand cDNA kit (Promega, Madison, WI, USA). $5 \mu \mathrm{l}$ of total cDNA of each sample was amplified in final volume of $25 \mu \mathrm{l}$ of reaction mixture containing Platinum ${ }^{\circ}$ SYBR $^{\circ}$ Green qPCR SuperMix-UDG ready-to-use reaction cocktail and specific primers for Bmp2, Bmp4, Bmp7, Bmpr1a, Bmpr1b, Bmpr2 or $\beta$-actin using the ABI StepOne Plus system (all from Applied Biosystems, CA, USA) (Table 1). Cycling conditions were: denaturation at $95^{\circ} \mathrm{C}$ for $10 \mathrm{mi}-$ nutes, 45 cycles at $95^{\circ} \mathrm{C}$ for 20 seconds, optimal annealing temperature (Table 1 ) for 20 seconds, $72^{\circ} \mathrm{C}$ for 30 seconds and finally at $60-95^{\circ} \mathrm{C}$ with a heating rate of $0.1^{\circ} \mathrm{C} /$ second. The expression of target gene was normalized to that of $\beta$-actin gene. Relative gene expression was calculated with the $2^{-\triangle \mathrm{CT}}$ formula. The mRNA expression of BMPs and BMP receptors were the results of 6 rats from each group.

\section{Western blotting (WB)}

The cells were lysed and the concentration of total soluble protein was measured by BCA protein assay (Thermo Scientific, Rockford, IL). $50 \mu \mathrm{g}$ (for BMPs and BMP receptors) or $20 \mu \mathrm{g}$ (for pSmad 1/5/8 and total Smad 1/5/8) of protein was denatured, fractionated by electrophoresis on $12 \%(\mathrm{w} / \mathrm{v})$ sodium dodecyl sulfate (SDS)-polyacrylamide gel and then electrophoretically transferred onto nitrocellulose membrane (Pall, Ann Arbor, MI). The membrane was blocked with $5 \%(\mathrm{w} / \mathrm{v})$ non-fat dry milk in TBST solution (25 mM Trizma base (3.025 g), $125 \mathrm{mM} \mathrm{NaCl}$ (7.3 g), and $1 \mathrm{~mL}$ Tween-20, pH 7.6) and then incubated with primary antibody against BMP-2 (1:1000), BMP-4 (1:1000), BMP-7 (1:1000) (all from Abcam, Cambridge, UK), BM PR-IA (1: 500), BMPR-IB (1:200) (both from Santa Cruz Biotechnology, Santa Cruz, CA, USA), BMPR-II (1: 500; BD BioSciences, San Jose, California, USA), total Smad 1/ 5/8 (1:1000; Abcam, Cambridge, UK), pSmad 1/5/8 (1: 1000; Cell Signaling, Danvers, MA, USA) or $\beta$-actin antibody (1:1000, Santa Cruz Biotechnology, Santa Cruz, CA, USA). After washing, the membrane was incubated with horseradish peroxidase (HRP)-conjugated anti-goat secondary antibody (1:1000; Santa Cruz Biotechnology, Santa Cruz, CA, USA), HRP-conjugated anti-mouse secondary antibody (1:1000) or HRP-conjugated anti-rabbit secondary antibody (1:1000) (both from Merck Millipore, Darmstadt, Germany). Immunoreactive bands were detected by ECL reagents (Amersham Bioscience, Little Chalfont, UK). The same batch of samples was used for studying the expression

Table 1 The primer sequence, product size and annealing temperature of target genes for qRT-PCR

\begin{tabular}{|c|c|c|c|c|}
\hline Gene & Primer nucleotide sequence & Product size (bp) & Annealing temperature & Accession no. \\
\hline \multirow[t]{2}{*}{$\beta$-actin } & 5'-ATCGTGGGCCGCCCTAGGCA-3' (forward) & 243 & 52 & NM_031144 \\
\hline & 5'-TGGCCTTAGGGTTCAGAGGGG-3' (reverse) & & & \\
\hline \multirow[t]{2}{*}{ Bmp2 } & 5'-TAGTGACTITTGGCCACGACG-3' (forward) & 81 & 58 & NM_017178 \\
\hline & 5'-GCTTCCGCTGTTTGTGTTTG-3' (reverse) & & & \\
\hline \multirow[t]{2}{*}{ Bmp4 } & 5'-CATGGCTCGCGCCTCCTAGC-3' (forward) & 184 & 58 & NM_012827 \\
\hline & 5'- ATTCCGAGCGACGCACTGCC-3' (reverse) & & & \\
\hline \multirow[t]{2}{*}{ Bmp7 } & 5'- CAACCTAGTGGAGCACGACAAGGA-3' (reverse) & 213 & 60 & NM_001191856 \\
\hline & 5'- AGGTCGGACTCCCTGCCTGAGT-3' (reverse) & & & \\
\hline \multirow[t]{2}{*}{ Bmprla } & 5'-GCCACCCTGGACACCAGAGC-3' (forward) & 101 & 60 & NM_030849 \\
\hline & 5'-GCAGGCTTGCCTTGCGTG-3' (reverse) & & & \\
\hline \multirow[t]{2}{*}{ Bmprib } & 5'-CACCACGGAGGAAGCCAGC-3' (forward) & 239 & 60 & NM_001024259 \\
\hline & 5'-GGCACAGGCCGCTGACAGAC-3' (reverse) & & & \\
\hline \multirow[t]{2}{*}{ Bmpr2 } & 5'-GGTGCTGGTCTCACATTG-3' (forward) & 180 & 58 & NM_080407 \\
\hline & 5'-GAGGCGGACTGAGTGGTG-3' (reverse) & & & \\
\hline
\end{tabular}


of BMPs and BMPRs. Except for BMP-2, all the proteins used for the detection of BMPs and BMP receptors were loaded and ran within one week. Semi-quantitative image analyses of the protein expression of BMPs, BMP receptors, total Smad 1/5/8 and pSmad 1/5/8 were performed using the Adobe Photoshop software (Adobe Systems Incorporated, CA, USA, version 10.0). After thresholding, the region of interest (ROI) was selected by applying a rectangular box with size that was wide enough to cover the largest band among protein samples and with minimum height possible. The mean expression level of the target protein relative to $\beta$-actin was presented. As the expression of BMP-2 was studied at a different time compared to other BMPs and BMP receptors, different $\beta$-actin bands were used for normalization. Different $\beta$-actin bands of each independent experiment were used for normalization for the expression of total Smad 1/5/8 and pSmad 1/5/8. The Western analyses were the results of 3-4 rats from each group.

\section{Immunocytochemical staining (ICC)}

ICC was performed using UltraVision Quanto Detection System (Thermo Scientific, Kalamazoo, MI, USA). Briefly, the cells were fixed in $70 \%$ ethanol, quenched with $3 \%$ $\mathrm{H}_{2} \mathrm{O}_{2}$ in methanol for 20 minutes, blocked with Ultra $\mathrm{V}$ Block and incubated with rabbit anti-human pSmad 1/5/8 cross-reacted with rat pSmad 1/5/8 (1:200, Cell Signaling, Danvers, MA, USA) overnight at $4^{\circ} \mathrm{C}$. Primary antibody was replaced with blocking solution in the negative controls. After that, the cells were incubated with Primary Antibody Amplifier Quanto and then HRP Polymer Quanto for 10 minutes each at room temperature. Signal was visualized with DAB Quanto Chromogen/Substrate complex. The cells were rinsed in distilled water, counterstained in Harris hematoxylin, dehydrated through graded alcohol, and mounted with p-xylene-bis-pyridinium bromide (DPX) (Sigma Aldrich, St Louis, MO, USA). The cells were examined under light microscopy (DMRXA2, Leica Microsystems, Wetzlar GmbH, Germany). Representative results from 3 experiments were reported.

\section{Data analysis}

Quantitative and semi-quantitative data was shown in boxplots or time curve with mean and standard error (SE). For the boxplot, the lower, middle and upper boundaries of the box showed the $25^{\text {th }}, 50^{\text {th }}$ and $75^{\text {th }}$ percentile of the dataset. Observation with value that was more than 3 boxlength from the upper or lower edge of the box was shown as extreme value $(*)$ if existed. Observation with value that was between 1.5 to 3 box-length from the upper or lower edge of the box was shown as outlier (o) if existed. The largest and smallest observations in the dataset that were not outliers or extreme values were shown as whiskers. If there were no outliers and extreme values, the whiskers represented the maximum and the minimum observations of the dataset. The comparison of mRNA and protein expression of BMPs and BMP receptors between the TDSCs (CI) group and the TDSCs (HT) group was done using Mann-Whitney $U$ test. The comparison of the time series data of total Smad 1/5/8 and pSamd 1/5/8 between the TDSCs (CI) group and the TDSCs (HT) group was done using ANOVA fore repeated measures with time as the within-subjects factor and treatment group as the betweensubjects factor. All the data analysis was done using SPSS (SPSS Inc, Chicago, IL, version 16.0). p $<0.050$ was regarded as statistically significant.

\section{Results}

TDSCs (CI) expressed significantly higher mRNA levels of Bmp2 (2.2 fold, $\mathrm{p}=0.006)$, Bmp4 (1.7 fold, $\mathrm{p}=0.010$ ); Bmprla (1.9 fold, $\mathrm{p}=0.004$ ) and Bmprlb (1.6 fold, $\mathrm{p}=$ 0.016) but not Bmp7 ( $\mathrm{p}=0.670)$ and Bmpr2 ( $\mathrm{p}=0.873)$ compared to TDSCs (HT) (Figure 1). TDSCs (CI) also expressed significantly higher protein levels of BMP-2 (2.1 fold, $\mathrm{p}=0.0495$ ), BMP-4 (1.9 fold, $\mathrm{p}=0.021)$, BMP-7 (2.3 fold, $\mathrm{p}=0.021$ ), BMPR-IA ( 2.2 fold, $\mathrm{p}=0.0495$ ), BMPRIB (6.6 fold, $\mathrm{p}=0.034$ ) and BMPR-II (3.8 fold, $\mathrm{p}=0.034$ ) compared to TDSCs (HT) (Figures 2 and 3). There was higher expression of total Smad 1/5/8, but not pSmad 1/5/8 (both nuclear and cytosolic), in TDSCs (CI) compared to TDSCs (HT) (Figure 4A, B, C). Activated pSmad 1/5/8 was mainly located in the cell nucleus (Figure 5A, E). The addition of BMP-2 resulted in the increased expression and translocation of pSmad $1 / 5 / 8$ to the cell nucleus in both TDSCs (Figure 5B-D, F-H). The expression of total Smad $1 / 5 / 8$ and pSmad 1/5/8 (both nuclear and cytosolic) increased upon BMP-2 stimulation in both groups (Figure 4A, $\mathrm{B}, \mathrm{C}$ ). There was higher expression of pSmad 1/5/8 (both nuclear and cytosolic) and total Smad in TDSCs (CI) compared to TDSCs (HT) upon BMP-2 stimulation (pSmad 1/ 5/8: $\mathrm{p}=0.065$; total Smad: $\mathrm{p}=0.043$ ) (Figure 4B, C).

\section{Discussion}

While tissue stem/progenitor cells are essential for maintaining tissue homeostasis and repair, diseases may occur if the control of their renewal and differentiation goes aberrant [22]. We have previously hypothesized that TDSCs might undergo aberrant differentiation to non-tenocyte lineages, contributing to tissue metaplasia and failed healing in tendinopathy [22]. Our recent data supported this claim as TDSCs (CI) showed altered fate, with higher chondroosteogenic differentiation potential but lower tendonrelated marker expression compared to TDSCs (HT), which might contribute to pathological chondro-ossification and failed tendon healing in this animal model [18]. The mechanism(s) that contribute to the altered fate of TDSCs in the $\mathrm{CI}$ animal model is unclear. Both mechanical and biological factors might contribute to this. The present study was 


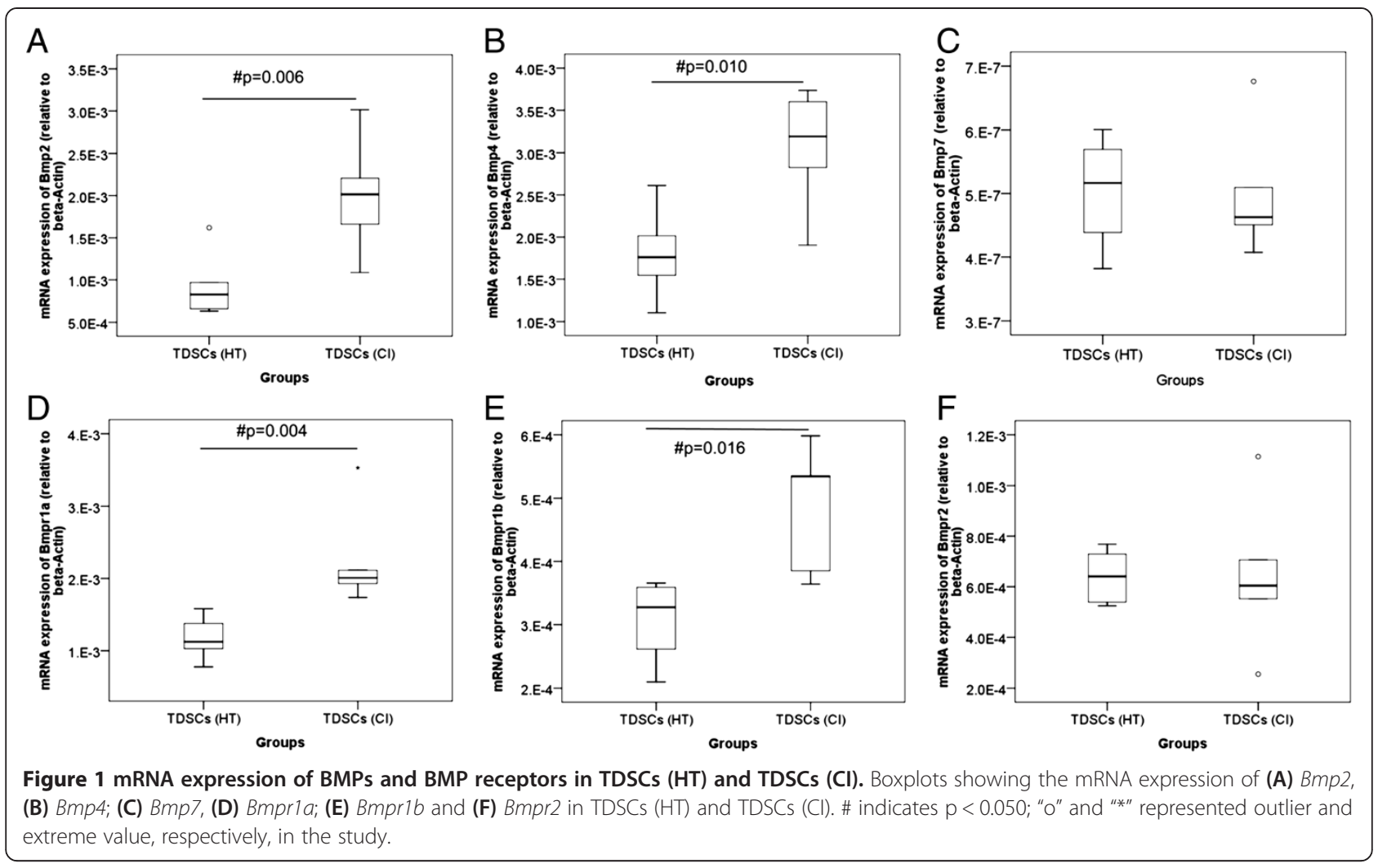

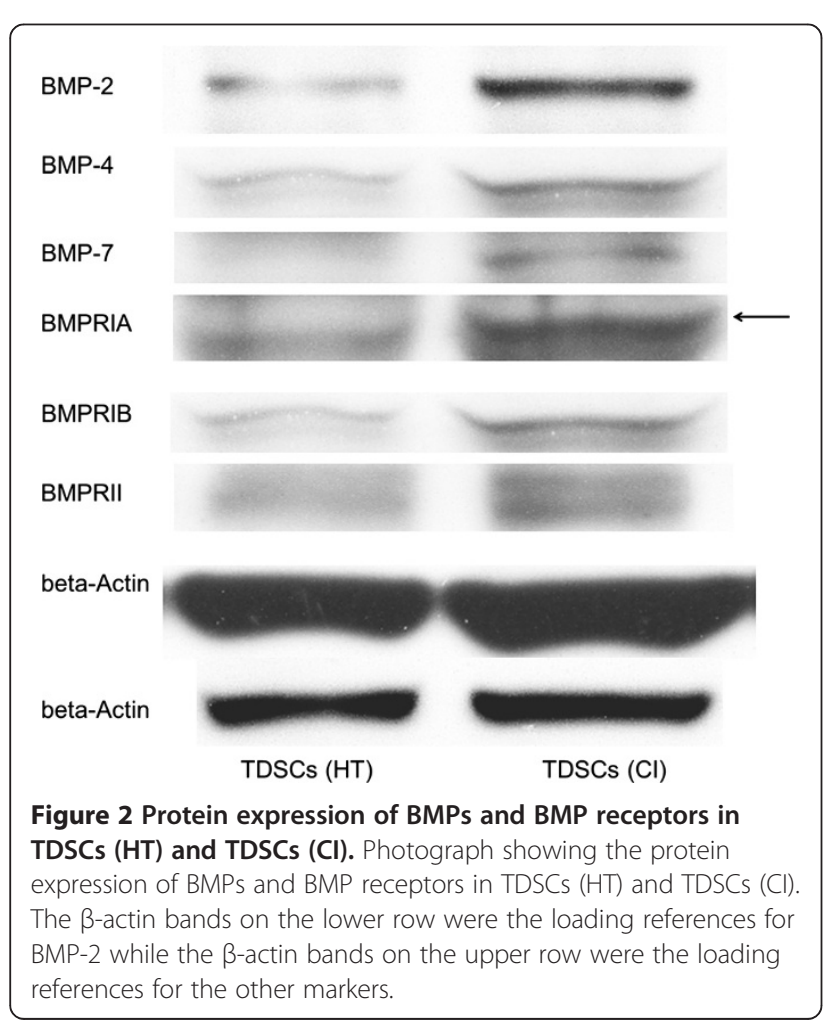

the first step to understand the mechanisms of altered fate of TDSC (CI) compared to TDSC (HT). In this study, we showed that the increased expression of BMPs and BMP receptors as well as elevated BMP/Smad sensitivity in TDSCs (CI) might contribute to the increased non-tenogenic differentiation potential and hence altered fate of these cells. While we detected increased protein expression of BMP-7 and BMPR-II in TDSCs (CI) compared to TDSCs (HT), we failed to detect increased expression of their corresponding mRNA. It might be due to different half-lives of protein and mRNA.

There was ectopic expression of BMP-2, BMP-4 and BMP-7 in clinical samples of tendinopathy $[9,10]$ and collagenase-induced tendon injury rat model [11]. No expression of BMP-2, BMP-4 and BMP-7 was observed in the intact tendons $[9,11]$. By exploring the mechanisms of the altered fate of TDSCs (CI), the present study added further support for the role of BMPs and the BMP/Smad signaling pathway in the pathogenesis of tendinopathy. We didn't examine the effect of BMP-2 on the non-tenogenic differentiation of TDSC (CI) and TDSC (HT) in this study because our previous results have already demonstrated that TDSC (CI) showed higher spontaneous chondro-osteogenesis in basal medium compared to TDSC (HT) [18]. Moreover, BMP-2 alone in basal culture medium was sufficient to induce non-tenogenic differentiation of TDSCs [13]. Similar to our findings, 

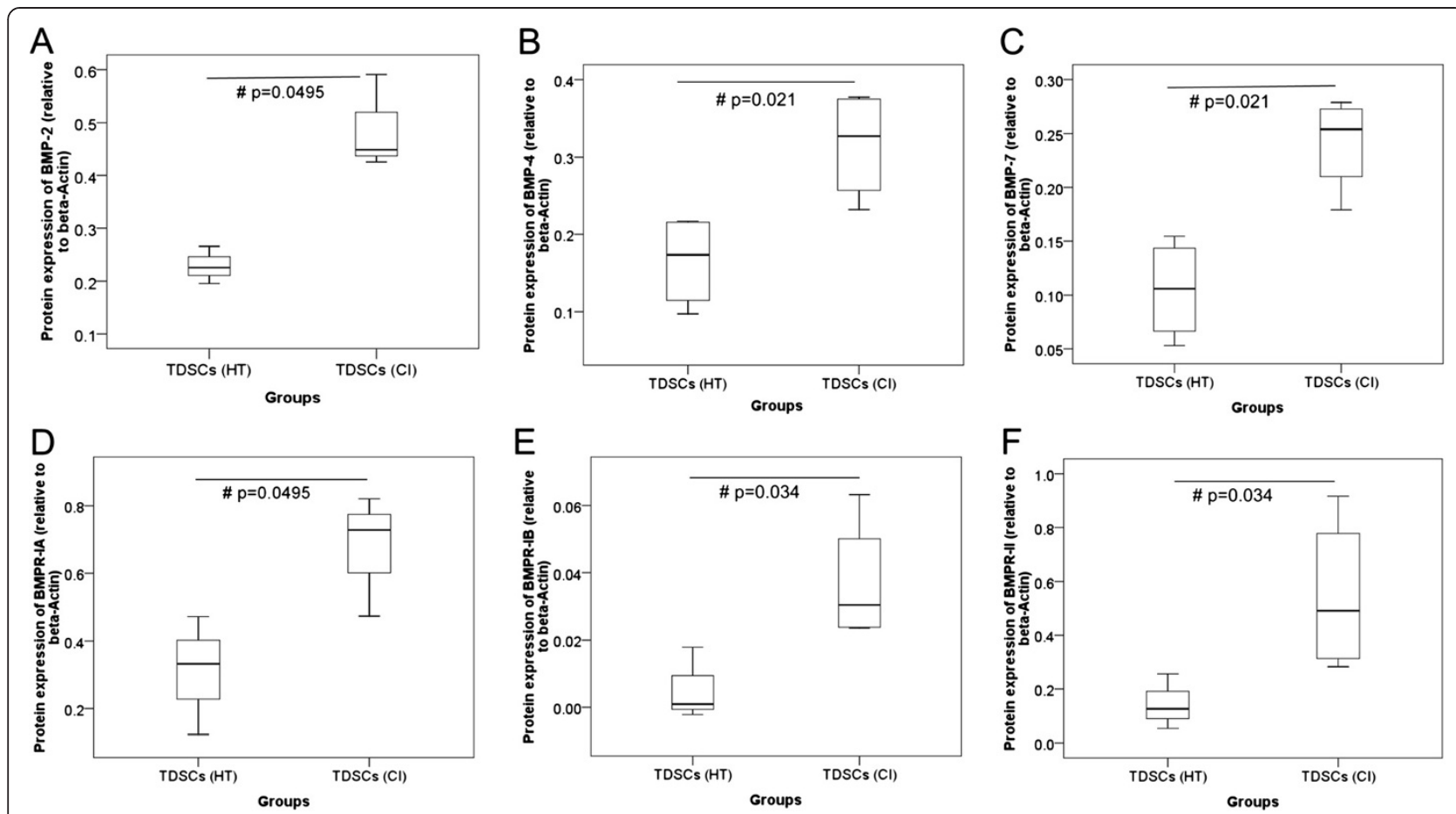

Figure 3 Semi-quantitative analysis of BMPs and BMP receptors in TDSCs (HT) and TDSCs (Cl). Boxplots showing the semi-quantitative analysis of the protein band intensity relative to $\beta$-actin for (A) BMP-2, (B) BMP-4, (C) BMP-7, (D) BMPR-IA, (E) BMPR-IB and (F) BMPR-II in TDSCS $(\mathrm{HT})$ and TDSCS (Cl). \# indicates $\mathrm{p}<0.050$.

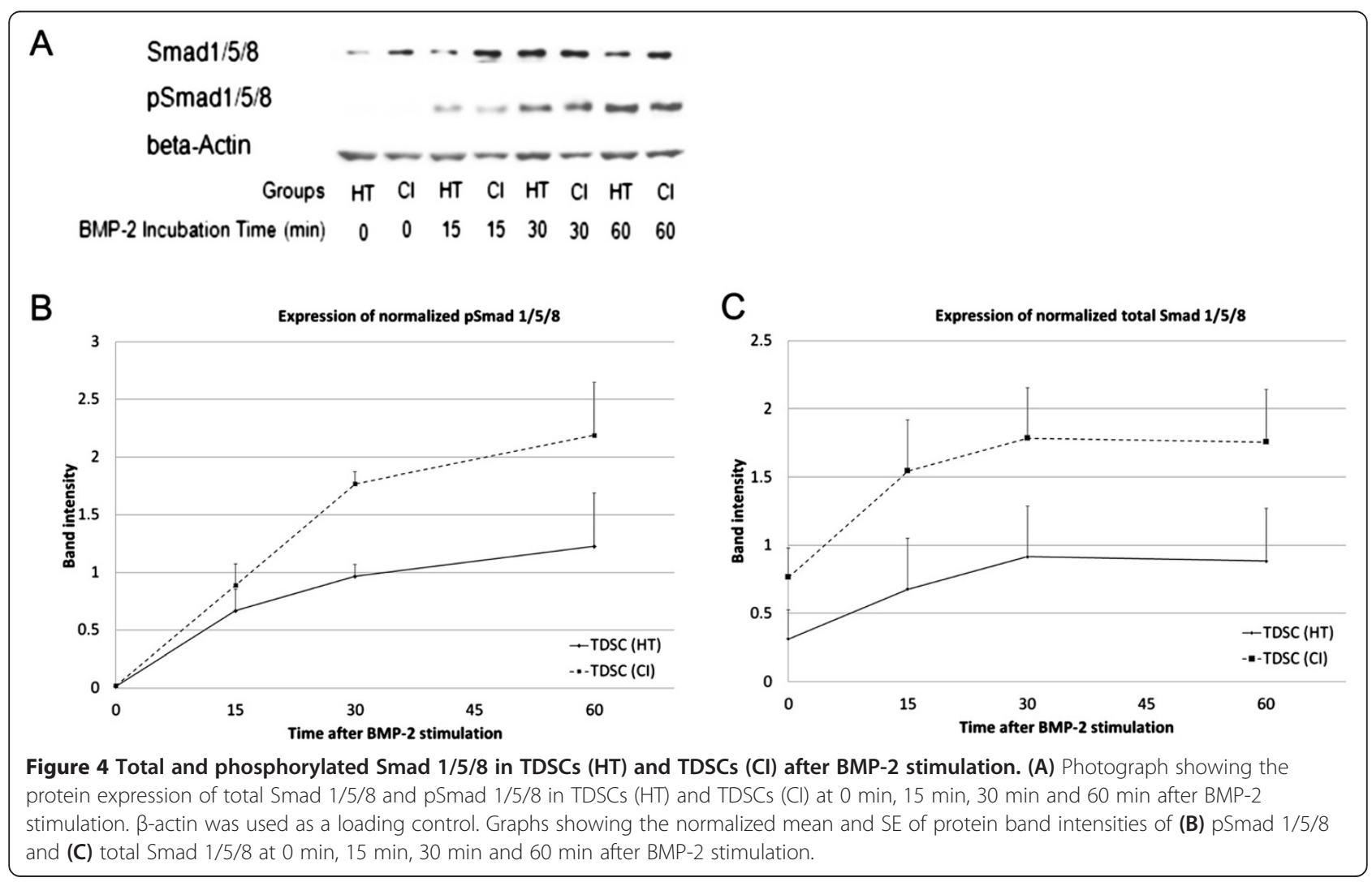




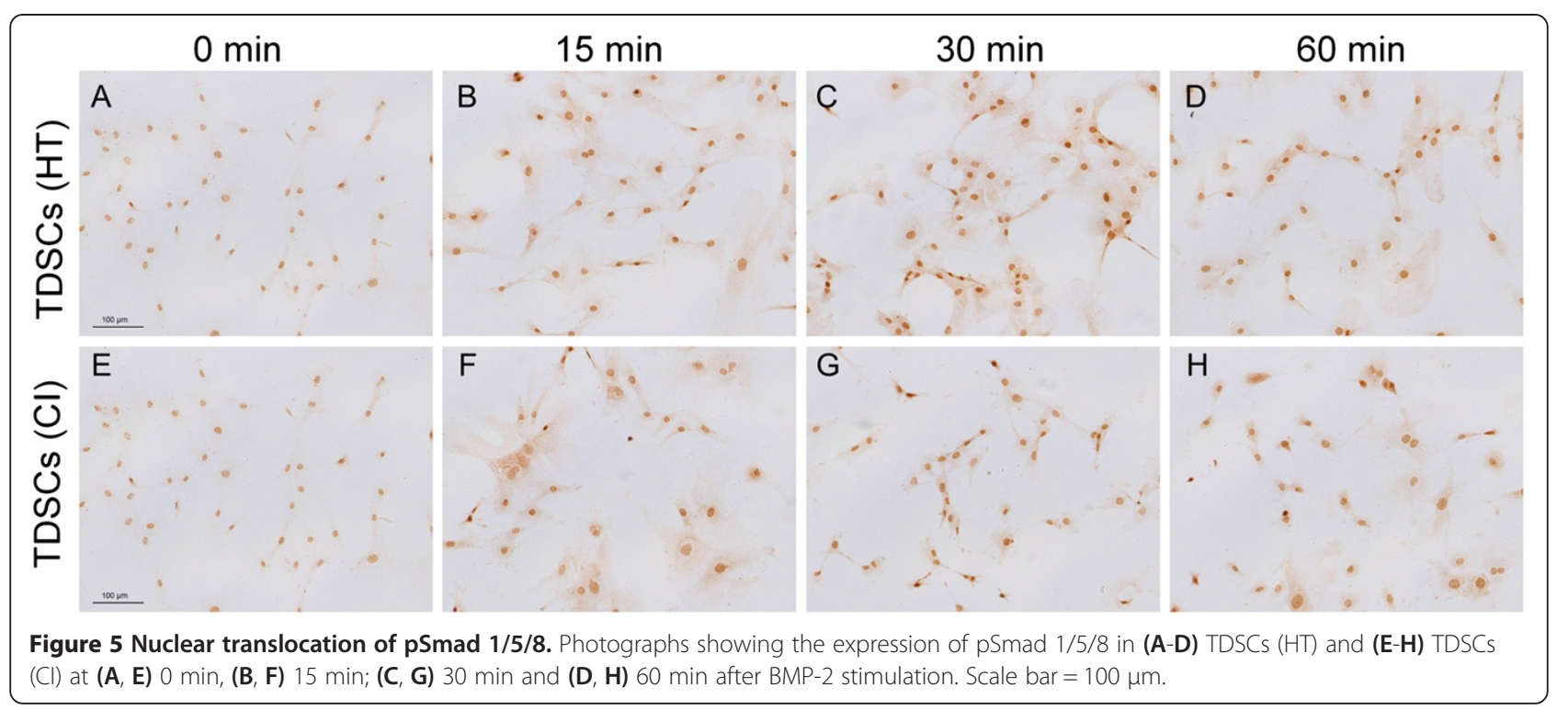

tendon stem / progenitor cells (TSPCs) isolated from disorganized, calcified tendons of biglycan and fibromodulin double knock-out mice were more sensitive to BMP-2 stimulation with increased phosphorylation of Smad 1/5/8 and more abundant nuclear localization of phosphorylated Smad1/5/8 than that in wild-type cells [21]. Fibrodysplasia Ossificans Progressiva (FOP) is characterized by progressive heterotropic ossification. Connective tissue progenitor cells from the discarded primary teeth of patients with FOP showed more rapid differentiation to an osteogenic phenotype as well as higher basal and stimulated BMP signaling compared to those in the control cells [23]. Of note was that both the transgenic animal model [21] and the FOP patients [23] have genetic mutations while the animal model that we used in this study was established using biological agents after birth.

The sources of BMPs after collagenase-induced tendon injury were unknown. The change of tendon loading as a result of tendon overuse has been suggested as one of the etiological factors of tendinopathy [24]. Our previous study showed that TDSCs isolated from the healthy animals produced BMP-2 in response to repetitive cyclic tensile loading [14]. Whether tendon overuse would lead to increased BMP sensitivity of TDSCs as reported in this study and how the increased expression of BMPs and BMP receptors would feedback and modulate the sensitivity of TDSCs to mechanical loading requires further research. Besides mechanical loading, the sources of BMPs might come from other cell types such as tenocytes and/or release from the extracellular matrix after collagenase-induced tendon injury. Further research is needed to confirm our speculation.

This study has some limitations. First, the pathological concentration of BMP-2 in tendinopathy was not known.
The dose used in this study was chosen based on our previous study [13] and another report [21]. The use of pathological concentration of BMP-2 in tendinopathy for TDSC stimulation in vitro would yield more clinically relevant data. This study was our first step to understand the mechanisms of non-tenogenic differentiation and hence altered fate of TDSCs (CI). A more fundamental question of why TDSCs (CI) have higher BMP and BMPR expression as well as elevated BMP/Smad sensitivity remains unanswered. We did not examine the fate of TDSCs (CI) by inhibiting the BMP/Smad signaling pathway in this study, the results of which would provide further support of BMPs and the BMP/Smad signaling pathway in regulating the fate of TDSCs (CI) and might shed lights on the pathogenesis and treatment of tendinopathy. Further study is required.

\section{Conclusions}

In conclusion, except for the mRNA levels of $B m p 7$ and Bmpr2, TDSCs isolated from the collagenase-induced patellar tendon injury rat model expressed higher mRNA and protein levels of BMPs (BMP-2, BMP-4, BMP-7) and BMP receptors (BMPR-IA, BMPR-IB, BMPR-II) compared to the TDSCs isolated from the healthy animals. They were also more sensitive to BMP-2 stimulation compared to the TDSCs isolated from the healthy animals. The sensitization of the BMP/Smad pathway might account for the higher non-tenogenic differentiation potential and hence altered fate of TDSCs in the collagenase-induced tendon injury rat model. It also provided further support of BMPs and the BMP/Smad signaling pathway in the pathogenesis of tendinopathy. 


\section{Competing interests}

The authors declare that they have no competing interests.

\section{Authors' contributions}

PPYL conceived of the study, designed the study, analyzed the data and drafted the manuscript. YMW performed the experiments and analyzed the data. Both authors read and approved the final manuscript.

\section{Acknowledgements}

This work was supported by equipment/resources donated by the Hong Kong Jockey Club Charities Trust.

\section{Author details}

${ }^{1}$ Headquarter, Hosptial Authority, 9/F, Rumsey Street Multi-Storey Carpark Building, Sheung Wan, Hong Kong SAR, China. ${ }^{2}$ Department of Orthopaedics and Traumatology, Faculty of Medicine, The Chinese University of Hong Kong, Hong Kong SAR, China. ${ }^{3}$ The Hong Kong Jockey Club Sports Medicine and Health Sciences Centre, Faculty of Medicine, The Chinese University of Hong Kong, Hong Kong SAR, China.

Received: 23 March 2013 Accepted: 20 August 2013

Published: 21 August 2013

\section{References}

1. Rolf C, Movin T: Etiology, histopathology, and outcome of surgery in achillodynia. Foot Ankle Int 1997, 18(9):565-569.

2. Riley GP, Harrall RL, Constant CR, Cawston TE, Hazleman BL: Prevalence and possible pathological significance of calcium phosphate salt accumulation in tendon matrix degeneration. Ann Rheum Dis 1996, 55:109-115.

3. Rolf CG, Fu BS, Pau A, Wang W, Chan B: Increased cell proliferation and associated expression of PDGFRbeta causing hypercellularity in patellar tendinosis. Rheumatology (Oxford) 2001, 40:256-261.

4. Kannus P, Jozsa L: Histopathological changes preceding spontaneous rupture of a tendon. A controlled study of 891 patients. J Bone Joint Surg Am 1991, 73:1507-1525.

5. Maffulli N, Reaper J, Ewen SWB, Waterston SW, Barrass V: Chondral metaplasia in calcific tendinopathy of the Achilles tendon. Clin J Sport Med 2006, 16:329-334.

6. Larsson ME, Käll I, Nilsson-Helander K: Treatment of patellar tendinopathy - a systematic review of randomized controlled trials. Knee Surg Sports Traumatol Arthrosc 2012, 20(8):1632-1646.

7. Kearney R, Costa ML: Insertional Achilles tendinopathy management: a systematic review. Foot Ankle Int 2010, 31(8):689-694.

8. Rees JD, Maffulli N, Cook J: Management of tendinopathy. Am J Sports Med 2009, 37:1855-1867.

9. Rui YF, Lui PP, Rolf CG, Wong YM, Lee YW, Chan KM: Expression of chondro-osteogenic BMPs in clinical samples of patellar tendinopathy. Knee Surg Sports Traumatol Arthrosc 2011, 20:1409-1417.

10. Neuwirth J, Fuhrmann RAE, Veit A, Aurich M, Stonans I, Trommer T, Hortschansky P, Chubinskaya S, Mollenhauer JA: Expression of bioactive bone morphogenetic proteins in the subacromial bursa of patients with chronic degeneration of the rotator cuff. Arthritis Res Ther 2006, 8:R92.

11. Yee Lui PP, Wong YM, Rui YF, Lee YW, Chan LS, Chan KM: Expression of chondro-osteogenic BMPs in ossified failed tendon healing model of tendinopathy. J Orthop Res 2011, 29:816-821.

12. Hata K, Nishimura R, Ikeda F, Yamashita K, Matsubara T, Nokubi T, Yoneda T: Differential roles of Smad1 and p38 kinase in regulation of peroxisome proliferator-activating receptor gamma during bone morphogenetic protein 2-indcued adipogenesis. Mol Biol Cell 2003, 14:545-555.

13. Rui YF, Lui PPY, Wong YM, Tan Q, Chan KM: BMP-2 stimulated nontenogenic differentiation and promoted proteoglycan deposition of tendon-derived stem cells (TDSCs) in vitro. J Orthop Res 2013, 31:746-753.

14. Rui YF, Lui PP, Ni M, Chan LS, Lee YW, Chan KM: Mechanical loading increased BMP-2 expression which promoted osteogenic differentiation of tendon-derived stem cells. J Orthop Res 2011, 29:390-396.

15. Shea CM, Edgar CM, Einhorn TA, Gerstenfeld LC: BMP treatment of C3H10T1/2 mesenchymal stem cells induces both chondrogenesis and osteogenesis. J Cell Biochem 2003, 90:1112-1127.

16. Toh WS, Yang Z, Liu H, Heng BC, Lee EH, Cao T: Effects of culture conditions and bone morphogenetic protein 2 on extent of chondrogenesis from human embryonic stem cells. Stem Cells 2007, 25:950-960.

17. de Mos M, Koevoet W, van Schie HT, Kops N, Jahr H, Verhaar JA, van Osch GJ: In vitro model to study chondrogenic differentiation in tendinopathy. Am J Sports Med 2009, 37:1214-1222.

18. Rui YF, Lui PPY, Wong YM, Tan Q, Chan KM: Altered fate of tendon-derived stem cells (TDSCs) isolated from a failed tendon healing animal model of tendinopathy. Stem Cells Dev 2013, 22(7):1076-1085.

19. Lui PPY, Fu SC, Chan LS, Hung LK, Chan KM: Chondrocyte phenotype and ectopic ossification in collagenase-induced tendon degeneration. J Histochem Cytochem 2009, 57:91-100.

20. Rui YF, Lui PP, Li G, Fu SC, Lee YW, Chan KM: Isolation and characterization of multipotent rat tendon-derived stem cells. Tissue Eng Part A 2010, 16:1549-1558.

21. Bi Y, Ehirchiou D, Kilts TM, Inkson CA, Embree MC, Sonoyama W, Li L, Leet Al, Seo BM, Zhang L, Shi S, Young MF: Identification of tendon stem/progenitor cells and the role of the extracellular matrix in their niche. Nat Med 2007 13:1219-1227.

22. Lui PP, Chan K: Tendon-derived stem cells (TDSCs): From basic science to potential roles in tendon pathology and tissue engineering applications. Stem Cell Rev 2011, 7:883-897.

23. Billings PC, Fiori JL, Bentwood JL, O'Connell MP, Jiao X, Nussbaum B, Caron RJ, Shore EM, Kaplan FS: Dysregulated BMP signaling and enhanced osteogenic differentiation of connective tissue progenitor cells from patients with fibrodysplasia ossificans progressiva (FOP). J Bone Miner Res 2008, 23:305-313.

24. Cook JL, Purdam C: Is compressive load a factor in the development of tendionpathy? Br J Sports Med 2012, 46(3):163-168.

doi:10.1186/1471-2474-14-248

Cite this article as: Lui and Wong: Higher BMP/Smad sensitivity of tendon-derived stem cells (TDSCs) isolated from the collagenase-induced tendon injury model: possible mechanism for their altered fate in vitro. BMC Musculoskeletal Disorders 2013 14:248.

\section{Submit your next manuscript to BioMed Central and take full advantage of:}

- Convenient online submission

- Thorough peer review

- No space constraints or color figure charges

- Immediate publication on acceptance

- Inclusion in PubMed, CAS, Scopus and Google Scholar

- Research which is freely available for redistribution 